Bulletin of the Section of Logic

Volume 46:3/4 (2017), pp. 219-232

http://dx.doi.org/10.18778/0138-0680.46.3.4.04

Marek Nowak

\title{
A SYNTACTIC APPROACH TO CLOSURE OPERATION
}

\begin{abstract}
In the paper, tracing the traditional Hilbert-style syntactic account of logics, a syntactic characteristic of a closure operation defined on a complete lattice follows. The approach is based on observation that the role of rule of inference for a given consequence operation may be played by an ordinary binary relation on the complete lattice on which the closure operation is defined.
\end{abstract}

Keywords: closure operation, closure system, rule of inference

\section{Preliminaries}

The paper deals mainly with closure operations defined on a complete lattice. Given a complete lattice $(A, \leq)$, any mapping $C: A \longrightarrow A$ such that for each $a \in A, a \leq C(a), C(C(a)) \leq C(a)$ and $C$ is monotone: $a \leq b \Rightarrow C(a) \leq C(b)$, is called a closure operation defined on $(A, \leq)$. Any subset $B \subseteq A$ is said to be a closure system of the lattice $(A, \leq)$, if for each $X \subseteq B, \inf _{A} X \in B$. Given a closure operation $C$ on $(A, \leq)$, the set of all its fixed points called closed elements: $\{a \in A: a=C(a)\}$, is a closure system of $(A, \leq)$. Conversely, given a closure system $B$ of $(A, \leq)$, the map $C: A \longrightarrow A$ defined by $C(a)=\inf _{A}\{x \in B: a \leq x\}$, is a closure operation on $(A, \leq)$. The closure system $B$ is just the set of all its closed elements. On the other hand, the closure system of all closed elements of a given closure operation $C$ defines, in that way, just the operation $C$. Thus, there is a one to one correspondence between the class of all closure operations and of all closure systems of $(A, \leq)$ (in fact, it is a dual isomorphism between the respective complete lattices of all closure operations and closure systems). Any closure system $B$ of $(A, \leq)$ forms a complete lattice with respect to the order $\leq \operatorname{such}_{\text {that }} \inf _{B} X=\inf _{A} X$ and 
$\sup _{B} X=C\left(\sup _{A} X\right)$, for each $X \subseteq B$, where $C$ is the closure operation corresponding to closure system $B$. Given a subset $X$ of $A$, there exists the least closure system $B$ of $(A, \leq)$ such that $X \subseteq B$. It is called a closure system generated by $X$ and shall be denoted by $[X]$. It is simply the intersection of all the closure systems of $(A, \leq)$ containing $X$ and is of the form: $[X]=\left\{\inf _{A} Y: Y \subseteq X\right\}$. The closure operation $C$ corresponding to closure system $[X]$ is expressed by $C(a)=\inf _{A}\{x \in X: a \leq x\}$, any $a \in A$. Any closure system $\mathcal{B}$ of a complete lattice $(\wp(L), \subseteq)$ of all subsets of a given set $L$ is usually called a closure system over $L$. The corresponding closure operation $C: \wp(L) \longrightarrow \wp(L)$ assigns to each $X \subseteq L$ the smallest element of $\mathcal{B}$ containing $X: C(X)=\bigcap\{Y \in \mathcal{B}: X \subseteq Y\}$.

An interior operation, an interior system and open element are the dual concepts with respect to closure ones.

Some elements of the theory of Galois connections shall be used in the paper. The general theory is to be found for example in [1], [2], [4]. Given the posets $\left(A, \leq_{A}\right),\left(B, \leq_{B}\right)$, any pair of mappings $f: A \longrightarrow B, g: B \longrightarrow$ $A$ such that for each $a \in A, b \in B: b \leq_{B} f(a)$ iff $a \leq_{A} g(b)$, is called an antimonotone Galois connection for those posets. Equivalently, such a Galois connection $(f, g)$ fulfils the following conditions: $a \leq_{A} g(f(a)), b \leq_{B}$ $f(g(b))$ for any $a \in A, b \in B$ and $f, g$ are antimonotone. Given Galois connection $(f, g)$ for the posets $\left(A, \leq_{A}\right),\left(B, \leq_{B}\right)$, the ranges $f[A], g[B]$ of the mappings $f$ and $g$ are the sets of all closed elements with respect to the following closure operations $C_{2}, C_{1}$, respectively: for each $a \in A, b \in$ $B, C_{2}(b)=f(g(b)), C_{1}(a)=g(f(a))$. Since for each $a \in g[B], b \in f[A]$ : $g(f(a))=a, f(g(b))=b$ and moreover, for any $a_{1}, a_{2} \in g[B]: a_{1} \leq_{A} a_{2}$ iff $f\left(a_{2}\right) \leq_{B} f\left(a_{1}\right)$, so the complete lattices $\left(g[B], \leq_{A}\right),\left(f[A], \leq_{B}\right)$ are dually isomorphic (with $f$ being a dual isomorphism).

Especially important is so-called archetypal Galois connection (cf. for example, [3]). Having the sets $A, B$ and a binary relation $\rho \subseteq A \times B$, an archetypal antimonotone Galois connection $(f, g)$ is defined for the complete lattices $(\wp(A), \subseteq),(\wp(B), \subseteq)$ in the following way. For any $X \subseteq A$ and $b \in B, b \in f(X)$ iff $\forall a \in X a \rho b$. On the other hand, for each $Y \subseteq B$ and $a \in A, a \in g(Y)$ iff $\forall b \in Y a \rho b$.

In turn, a pair $f: A \longrightarrow B, g: B \longrightarrow A$ such that for each $a \in$ $A, b \in B: b \leq_{B} f(a)$ iff $g(b) \leq_{A} a$, is called a monotone Galois connection or a residuated pair of mappings for the posets $\left(A, \leq_{A}\right)$, $\left(B, \leq_{B}\right)$. Equivalently, a monotone Galois connection $(f, g)$ fulfils the fol- 
lowing conditions: $g(f(a)) \leq_{A} a, b \leq_{B} f(g(b))$ for any $a \in A, b \in B$ and $f, g$ are monotone functions. Given a residuated pair $(f, g)$ for the posets $\left(A, \leq_{A}\right),\left(B, \leq_{B}\right)$, the ranges $f[A], g[B]$ are, respectively, the sets of all closed and open elements with respect to the following closure and interior operations $C, I$ : for each $a \in A, b \in B, C(b)=f(g(b)), I(a)=g(f(a))$. Since for each $a \in g[B], b \in f[A]: g(f(a))=a, f(g(b))=b$ and, moreover, for any $a_{1}, a_{2} \in g[B]: a_{1} \leq_{A} a_{2}$ iff $f\left(a_{1}\right) \leq_{B} f\left(a_{2}\right)$, so the complete lattices $\left(g[B], \leq_{A}\right),\left(f[A], \leq_{B}\right)$ are isomorphic (with $f$ being an isomorphism).

\section{Introduction}

Let us go along the lines of the traditional syntactic Hilbert-style approach to consequence operation (cf. for example [6], [7]). Let $L$ be a set of formulas of a given formal language and $\mathcal{R}$ be a set of rules of inference for $L$. Each rule of inference for $L$ is a binary relation $r \subseteq \wp(L) \times L$. Usually some additional conditions are put on a binary relation $r$ to make it a rule of inference, for example of the form: a set $Y$ is finite whenever $(Y, a) \in r$. However, it is convenient for our purposes to treat a rule of inference quite generally, as an arbitrary binary relation defined on the sets $\wp(L), L$. Now, such a set $\mathcal{R}$ of rules uniquely determines the family $\mathcal{C}$ of all subsets of $L$ which are closed on every rule $r \in \mathcal{R}$. A set $X \subseteq L$ is closed on a rule $r$ iff for any $(Y, a) \in r, a \in X$ whenever $Y \subseteq X$. The family $\mathcal{C}$ is a closure system over $L$ which in turn uniquely determines the closure operation $C: \wp(L) \longrightarrow \wp(L)$ called a consequence operation determined by the set of rules $\mathcal{R}$. That is, given an $X \subseteq L, C(X)$ is the least subset of $L$ containing $X$ and closed on each rule $r \in \mathcal{R}$. Notice that for any $X \subseteq L$ and any set $\mathcal{R}$ of rules of inference, $X$ is closed on each rule $r \in \mathcal{R}$ iff $X$ is closed on one rule: $\bigcup \mathcal{R}$. So instead of determining a consequence operation by a set of rules, a single rule of inference suffices: any rule of inference $r$ uniquely determines a closure system $\mathcal{C}_{r}$ of all sets closed on $r$ :

(1) $X \in \mathcal{C}_{r}$ iff for all $(Y, a) \in r(Y \subseteq X \Rightarrow a \in X)$.

The consequence operation determined by the rule $r$ is of the form: given $X \subseteq L, C_{r}(X)$ is the least element of the closure system $\mathcal{C}_{r}$ containing $X$.

In turn, a closure operation $C: \wp(L) \longrightarrow \wp(L)$ or the corresponding closure system $\mathcal{C}$ determine a rule of inference $r_{C}:(Y, a) \in r_{C}$ iff $\forall X \in$ $\mathcal{C}(Y \subseteq X \Rightarrow a \in X)$ iff $a \in C(Y)$. Moreover, for any family $\mathcal{B}$ of sets 
of formulas, the relation $r_{\mathcal{B}}$ defined by

(2) $(Y, a) \in r_{\mathcal{B}}$ iff for any $X \in \mathcal{B}(Y \subseteq X \Rightarrow a \in X)$,

coincides with $r_{C}$, where $C$ is the consequence operation determined by the closure system $[\mathcal{B}]$. So one can distinguish a pair of mappings:

(3) $\wp(\wp(L) \times L) \ni r \longmapsto \mathcal{C}_{r} \in \wp(\wp(L)), \wp(\wp(L)) \ni \mathcal{B} \longmapsto r_{\mathcal{B}} \in$ $\wp(\wp(L) \times L)$,

defined by (1) and (2). As it is easily seen, this pair of mappings is an archetypal Galois connection defined by a binary relation $S \subseteq(\wp(L) \times$ $L) \times \wp(L):((Y, a), X) \in S$ iff $(Y \subseteq X \Rightarrow a \in X)$. Here our point of interest is not the Galois connection but another pair of mappings:

(4) $\wp(\wp(L) \times L) \ni r \longmapsto C_{r} \in \operatorname{Cons}(L), \operatorname{Cons}(L) \ni C \longmapsto r_{C} \in$ $\wp(\wp(L) \times L)$,

joining two complete lattices, $(\wp(\wp(L) \times L), \subseteq),(\operatorname{Cons}(L), \leq)$, of all rules of inference for $L$ and of all consequence operations of $L$, respectively. The first mapping is a composition of two antimonotone mappings, $r \longmapsto \mathcal{C}_{r}$ defined by (1) and $\mathcal{C}_{r} \longmapsto C_{r}$. The second one is a composition of the antimonotone maps: $C \longmapsto \mathcal{C}$ and $\mathcal{C} \longmapsto r_{\mathcal{C}}$ defined by (2) (naturally, $\left.r_{\mathcal{C}}=r_{C}\right)$. So both mappings are monotone:

for any rules $r_{1}, r_{2}, r_{1} \subseteq r_{2} \Rightarrow C_{r_{1}} \leq C_{r_{2}}$,

for any consequence operations $C_{1}, C_{2}, C_{1} \leq C_{2} \Rightarrow r_{C_{1}} \subseteq r_{C_{2}}$.

Moreover, the following two facts are important. One of them is quite obvious, as it follows from the very definition of the consequence $C_{r}$. Another needs a proof.

For any rule of inference $r$ for $L, r \subseteq r_{C_{r}}$, that is, $r$ is a valid rule of $C_{r}$.

Given any consequence operation $C$ on $L, C_{r_{C}}=C$, (in equivalent form: any subset $X$ of $L$ is closed on the rule $r_{C}$ iff $X=C(X)$ ).

Proof. Given an $X \subseteq L$ and a consequence $C$ on $L$, the set $C(X)$ contains $X$ and is closed on the rule $r_{C}$. Now, let $Z \subseteq L$ contain $X$ and be closed on $r_{C}$. Suppose that $a \in C(X)$. Then $(X, a) \in r_{C}$ thus $a \in Z$. In this way, 
$C(X) \subseteq Z$, that is, $C(X)$ is the least set containing $X$ and closed on $r_{C}$, which means that $C(X)=C_{r_{C}}(X)$.

All four facts given above mean that the considered pair of mappings from (4), $C \longmapsto r_{C}, r \longmapsto C_{r}$, is a residuated pair. In this way, taking the fourth fact into account - namely, that the set of all open elements with respect to the interior operation induced by that residuated pair is just the set of all consequence operations on $L$ - one may state that

the complete lattice $(C o n s(L), \leq)$ of all consequence operations defined on a language $L$ is isomorphic to the complete lattice (Relcons $(L), \subseteq)$ of all rules of inference $r$ for $L$ such that $r=r_{C_{r}}$. The mapping $C \longmapsto r_{C}$ is that isomorphism.

It is not difficult to establish the conditions distinguishing the set Relcons $(L)$ as a subset of $\wp(\wp(L) \times L)$ :

for any rule of inference $r$ for $L, r=r_{C_{r}}$ iff $r$ is a so-called consequence relation on $L$, that is, the following conditions are satisfied for each $X, Y \subseteq L$ and $a \in L:$

(r1) $a \in X \Rightarrow(X, a) \in r$,

(r2) $X \subseteq Y \&(X, a) \in r \Rightarrow(Y, a) \in r$,

(r3) $(\{b \in L:(X, b) \in r\}, a) \in r \Rightarrow(X, a) \in r$.

Proof. $(\Rightarrow)$ : It is obvious that for any consequence $C$, the relation $r_{C}$ fulfils conditions (r1)-(r3).

$(\Leftarrow)$ : Consider any $r \subseteq \wp(L) \times L$ satisfying $(\mathrm{r} 1)-(\mathrm{r} 3)$. It is enough to show the inclusion $r_{C_{r}} \subseteq r$, i.e., the implication $a \in C_{r}(X) \Rightarrow(X, a) \in r$, for any $X \subseteq L$ and $a \in L$. So assume that $a \in C_{r}(X)$. Now let us prove that the set $\{b \in L:(X, b) \in r\}$ is closed on $r$. To this aim suppose that $(Y, d) \in r$ and $Y \subseteq\{b \in L:(X, b) \in r\}$. Hence and from (r2) it follows that $(\{b \in L:(X, b) \in r\}, d) \in r$. Therefore from $(\mathrm{r} 3),(X, d) \in r$, which means that $d \in\{b \in L:(X, b) \in r\}$. Furthermore, $X \subseteq\{b \in L:(X, b) \in r\}$ due to (r1). In this way, $C_{r}(X) \subseteq\{b \in L:(X, b) \in r\}$ by definition of $C_{r}$. Finally, $(X, a) \in r$ due to the assumption.

As one may see, we have arrived at a completely trivial point: identifying a consequence operation with a consequence relation. Just the syntactic approach without any restrictions put on the concept of rule of inference yields such a trivial effect. 
The purpose of the paper is to provide a similar syntactic approach to any closure operation defined on any complete lattice. It is clear that in such a case one cannot apply the concept of ordinary rule of inference, some counterpart of such a rule is needed. When the complete lattice $(\wp(L), \subseteq)$ of all subsets of the set of all formulas is considered, we propose to treat any binary relation $r \subseteq \wp(L) \times \wp(L)$ as such a counterpart. Then a closed set $B \subseteq L$ on $r$ may be defined as such that satisfies the condition: for any $(X, Y) \in r, X \subseteq B \Rightarrow Y \subseteq B$. The family $\mathcal{B}_{r}$ of all sets closed on $r$, that is, the family defined analogously as $\mathcal{C}_{r}$ in (1) by

(1') $B \in \mathcal{B}_{r}$ iff for all $(X, Y) \in r(X \subseteq B \Rightarrow Y \subseteq B)$,

forms a closure system over $L$, determining the closure operation, say, $C_{r}$ by $C_{r}(X)=\bigcap\left\{B \in \mathcal{B}_{r}: X \subseteq B\right\}$. This is just a non-trivial syntactic approach to closure operations defined on the lattice $(\wp(L), \subseteq)$. On the other hand, having any family $\mathcal{B}$ of sets of formulas (not necessary a closure system over $L$ ), one may define a binary relation (a "rule of inference") on $\wp(L)$, similarly as in (2), by

(2') $(X, Y) \in r_{\mathcal{B}}$ iff for any $B \in \mathcal{B}(X \subseteq B \Rightarrow Y \subseteq B)$.

The pair of mappings

(3') $\wp(L)^{2} \ni r \longmapsto \mathcal{B}_{r} \in \wp(\wp(L)), \wp(\wp(L)) \ni \mathcal{B} \longmapsto r_{\mathcal{B}} \in \wp(L)^{2}$,

now playing the role of (3), is an archetypal Galois connection determined by the ternary relation $R \subseteq \wp(L)^{3}:(X, Y, B) \in R$ iff $(X \subseteq B \Rightarrow Y \subseteq B)$.

Moreover, the relation $r_{\mathcal{B}}$ defined in (2'), may be treated, on the one hand, as a counterpart of the greatest valid rule of inference of the consequence $C$ determined by the closure system $[\mathcal{B}]$ :

(5) $(X, Y) \in r_{\mathcal{B}}$ iff $Y \subseteq \bigcap\{B \in \mathcal{B}: X \subseteq B\}$ iff $Y \subseteq C(X)$.

On the other hand, it may be conceived as a multiply-conclusion consequence relation of a special type. Here one may find an analogy to the concept of multiply-conclusion consequence relation $\vdash_{\mathcal{B}}$ introduced in [5] in the following way. Given a family $\mathcal{B} \subseteq \wp(L)$, for any $X, Y \subseteq L$,

(6) $X \vdash_{\mathcal{B}} Y$ iff for any $B \in \mathcal{B}(X \subseteq B \Rightarrow Y \cap B \neq \emptyset)$.

In [8] it was proved that when a family $\mathcal{B} \subseteq \wp(L)$ is a closure system over 
$L$, the consequence relation $\vdash_{\mathcal{B}}$ defined by (6) may be expressed by

(7) $X \vdash_{\mathcal{B}} Y$ iff $Y \cap C(X) \neq \emptyset$,

where $C$ is the consequence operation determined by the closure system $\mathcal{B}$. (7) means that given a set of premises, some of the conclusions of the relation $\vdash_{\mathcal{B}}$ are ordinary conclusions of consequence operation associated with the relation. So the relation $\vdash_{\mathcal{B}}$ has a disjunctive character. While according to (5), given a set of premises $X$, each of the conclusions of $r_{\mathcal{B}}$ is a conclusion of the consequence associated with $r_{\mathcal{B}}$. This means that the multiply-conclusion consequence relation $r_{\mathcal{B}}$ has a conjunctive character.

Now, adhering to the standard syntactic approach to consequence operation presented above, we can consider the following residuated pair of mappings being a counterpart of (4):

$\left(4^{\prime}\right) \wp\left(\wp(L)^{2}\right) \ni r \longmapsto C_{r} \in \operatorname{Cons}(L), \operatorname{Cons}(L) \ni C \longmapsto r_{C} \in$ $\wp\left(\wp(L)^{2}\right)$,

where $C_{r}$ is determined by the closure system $\mathcal{B}_{r}$ defined in (1'), and $r_{C}$ coincides with the relation $r_{\mathcal{B}}$ defined in $\left(2^{\prime}\right)$ or $(5)$, by the closure system $\mathcal{B}$ of all fixed points of the closure operation $C$. We are interested in a counterpart of the set of consequence relations Relcons $(L)$. However, we shall consider a more general case: the counterpart of this residuated pair defined on any complete lattice (in place of the lattice $(\wp(L), \subseteq)$ ). In order to find a counterpart of consequence relation from Relcons $(L)$, we shall proceed in three steps. First, we are going to provide a simple connection between all unary operations defined on a given complete lattice and binary relations of some kind defined on that lattice. Next, the connection will be confined to the set of all closure operations defined on that lattice. Finally, we shall be able to present a proper syntactic account of closure operations.

\section{On some correspondence between binary relations and unary operations defined on complete lattice}

Let $(A, \leq)$ be any complete lattice. Given a binary relation $r$ defined on the set $A$, including the lattice ordering $\leq$, and an element $x \in A$, let us put $[x)_{r},(x]_{r}$ for the sets $\{y \in A:(x, y) \in r\},\{y \in A:(y, x) \in r\}$, respectively. Let us consider the class $\operatorname{Rel}_{f u n} \subseteq \wp\left(A^{2}\right)$ of all the binary relations defined on $A$ fulfilling the following condition 
(fun) $\forall x \in A \exists z \in A,[x)_{r}=(z]_{\leq}$.

Notice the following obvious connection.

FACT 1 . For any complete lattice $(A, \leq)$ and any binary relation $r$ defined on $A$, condition (fun) is equivalent to

(fun') $\forall x \in A,[x)_{r}=\left(\sup [x)_{r}\right]_{\leq}$.

It is evident that for any mapping $c: A \longrightarrow A$, binary relation $r_{c} \subseteq A^{2}$ defined by $(x, y) \in r_{c}$ iff $y \leq c(x)$, satisfies the condition (fun) since, here, for any $x \in A,[x)_{r_{c}}=(c(x)]_{\leq}$. Conversely, given a binary relation $r \in$ $R e l_{\text {fun }}$, let us consider a mapping $c_{r}: A \longrightarrow A$ defined by $c_{r}(x)=\sup [x)_{r}$. Now take into account the complete lattice $\left(A^{A}, \leq\right)$ of all the mappings from $A$ to $A$ ordered by $\leq$ defined by $c_{1} \leq c_{2}$ iff $c_{1}(x) \leq c_{2}(x)$ for each $x \in A$.

Proposition 2. The complete lattices $\left(A^{A}, \leq\right),\left(\right.$ Rel $\left._{\text {fun }}, \subseteq\right)$, are isomorphic. The mapping $c \longmapsto r_{c}$ is the required isomorphism and the map $r \longmapsto c_{r}$ is the inverse one.

Proof. First, notice that given an arbitrary mapping $c: A \longrightarrow A, c_{r_{c}}=$ c. To show this fact, observe that for an $x \in A, c_{r_{c}}(x)=\sup [x)_{r_{c}}=$ $\sup (c(x)]_{\leq}=c(x)$. Moreover, given any binary relation $r \in \operatorname{Rel}_{f u n}$ we have $r_{c_{r}}=r$ since for any $x, y \in A,(x, y) \in r_{c_{r}}$ iff $y \leq c_{r}(x)$ iff $y \leq \sup [x)_{r}$ iff $(x, y) \in r$, due to Fact 1 and (fun'). Thus the mapping $c \longmapsto r_{c}$ is a bijection from $A^{A}$ onto $\operatorname{Rel}_{f u n}$. It remains to show that for any $c_{1}, c_{2} \in A^{A}, c_{1} \leq c_{2}$ iff $r_{c_{1}} \subseteq r_{c_{2}}$.

$(\Rightarrow)$ : Suppose that $c_{1} \leq c_{2}$ and let $(x, y) \in r_{c_{1}}$. Then $y \leq c_{1}(x) \leq$ $c_{2}(x)$, which implies $(x, y) \in r_{c_{2}}$.

$(\Leftarrow)$ : Assume that $r_{c_{1}} \subseteq r_{c_{2}}$ and let $x \in A$. From definition of $r_{c_{2}}$, it follows that $c_{1}(x) \leq c_{2}(x)$ iff $\left(x, c_{1}(x)\right) \in r_{c_{2}}$. Analogously, $c_{1}(x) \leq c_{1}(x)$ iff $\left(x, c_{1}(x)\right) \in r_{c_{1}}$. Hence $\left(x, c_{1}(x)\right) \in r_{c_{1}}$, so taking the assumption into account we have $\left(x, c_{1}(x)\right) \in r_{c_{2}}$. Therefore, $c_{1}(x) \leq c_{2}(x)$.

In the complete lattice $\left(\operatorname{Rel}_{f u n}, \subseteq\right)$ for any nonempty family $\mathcal{R} \subseteq$ $\operatorname{Rel}_{\text {fun }}$ we have $\inf \mathcal{R}=\bigcap \mathcal{R}$ and $\inf \emptyset=A^{2}$. To show it, notice that inf $\mathcal{R}$ corresponds by the isomorphism to $\inf \left\{c_{r}: r \in \mathcal{R}\right\}$ in the complete lattice $\left(A^{A}, \leq\right)$, that is, $(x, y) \in \inf \mathcal{R}$ iff $y \leq c(x)$, where $c=\inf \left\{c_{r}: r \in \mathcal{R}\right\}$. So we have $(x, y) \in \inf \mathcal{R}$ iff $y \leq\left(\inf \left\{c_{r}: r \in \mathcal{R}\right\}\right)(x)$ iff $y \leq \inf \left\{c_{r}(x)\right.$ : 
$r \in \mathcal{R}\} \quad$ iff for each $r \in \mathcal{R}, y \leq c_{r}(x)$ iff for each $r \in \mathcal{R},(x, y) \in r$ iff $(x, y) \in \bigcap \mathcal{R}$.

\section{Correspondence between binary relations and closure operations}

Now we confine ourselves to some classes of the mappings from $A^{A}$ and the corresponding families of binary relations. First, let us consider the class $E x(A)$ of all mappings $c: A \longrightarrow A$ satisfying the expansion condition: $x \leq c(x)$ for any $x \in A$. As it is well-known, the poset $(E x(A), \leq)$ is a complete sublattice of the complete lattice $\left(A^{A}, \leq\right)$.

FACT 3. The correspondence Ex $(A) \ni c \longmapsto r_{c}$ is an isomorphism of the complete lattices $(\operatorname{Ex}(A), \leq)$ and $\left(\operatorname{Refl}_{f u n}, \subseteq\right)$ of all reflexive relations on A fulfilling condition (fun).

Proof. Let us restrict the isomorphism $A^{A} \ni c \longmapsto r_{c}$ to the class $\operatorname{Ex}(A)$. When $c \in E x(A), r_{c}$ is obviously reflexive on $A$. On the other hand, if $r \in \operatorname{Rel}_{\text {fun }}$ is reflexive, for a given $x \in A, x \leq \sup [x)_{r}$. Hence the corresponding map $c_{r}$ fulfils the condition: $x \leq c_{r}(x)$ for all $x \in A$.

Now let $\operatorname{Mon}(A)$ be the class of all monotone mappings from $A$ to $A$. As for $\operatorname{Ex}(A)$, the poset $(\operatorname{Mon}(A), \leq)$ is a complete sublattice of the complete lattice $\left(A^{A}, \leq\right)$. Moreover, let $\operatorname{Re}_{\text {fun }}^{\text {mon }}$ be the class of all binary relations satisfying the conditions (fun) and

$$
\text { (mon) }(x, z) \in r \& x \leq y \Rightarrow(y, z) \in r, \text { for all } x, y, z \in A .
$$

or equivalently,

$$
\text { (mon') } x \leq y \Rightarrow[x)_{r} \subseteq[y)_{r}, \text { for all } x, y \in A .
$$

FACT 4. The correspondence Mon $(A) \ni c \longmapsto r_{c}$ is an isomorphism of the complete lattices $(\operatorname{Mon}(A), \leq)$ and $\left(\right.$ Rel $\left._{\text {fun }}^{\text {mon }}, \subseteq\right)$.

Proof. Let us restrict the isomorphism $A^{A} \ni c \longmapsto r_{c}$ to the class $\operatorname{Mon}(A)$. Suppose that a map $c: A \longrightarrow A$ satisfies the condition: $x \leq$ $y \Rightarrow c(x) \leq c(y)$, for all $x, y \in A$. Then, obviously, the relation $r_{c}$ fulfils condition (mon). Conversely, when a given $r \in \operatorname{Rel}_{\text {fun }}$ satisfies (mon'), the map $c_{r}$ is monotone: suppose $x \leq y$; then obviously by (mon'), $\sup [x)_{r} \leq \sup [y)_{r}$ so $c_{r}(x) \leq c_{r}(y)$. 
Now, let us consider the class $R T(A)$ of all monotone mappings $c$ : $A \longrightarrow A$ such that for any $x \in A, c(c(x)) \leq c(x) . R T(A)$ does not form a complete sublattice of $\left(A^{A}, \leq\right)$, however, for any its subclass $\mathcal{C}$ the infimum of $\mathcal{C}$ in the complete lattice $\left(A^{A}, \leq\right)$ belongs to $R T(A)$. In this way, one can consider $(R T(A), \leq)$ as a complete lattice.

FACT 5. The correspondence $R T(A) \ni c \longmapsto r_{c}$ is an isomorphism of the complete lattices $(R T(A), \leq)$ and $\left(\right.$ Trans $\left._{\text {fun }}^{\text {mon }}, \subseteq\right)$ of all transitive relations $r$ from Rel fun $_{\text {satisfying (mon). }}$

Proof. The proof goes analogously to the proofs of Facts 3 and 4. Having restricted the isomorphism to the domain $R T(A)$, let us check whether given $c \in R T(A), r_{c}$ is transitive. Suppose that $(x, y),(y, z) \in r_{c}$. Then $y \leq c(x)$ and $z \leq c(y)$. So $c(y) \leq c(c(x)) \leq c(x)$, thus $z \leq c(x)$, which means that $(x, z) \in r_{c}$. Moreover, $r_{c}$ satisfies (mon) (cf. the proof of Fact 4). Therefore, $r_{c} \in$ Trans $_{\text {fun }}^{\text {mon }}$. Conversely, suppose that $r \in$ Trans $_{\text {fun }}^{\text {mon }}$. Then the map $c_{r}$ is monotone due to the proof of Fact 4. Furthermore, we have $\left(x, \sup [x)_{r}\right) \in r$ from (fun'). Now we show that $\left[\sup [x)_{r}\right)_{r} \subseteq$ $[x)_{r}$. To this aim suppose that $\left(\sup [x)_{r}, y\right) \in r$. Then, from transitivity of $r$, it follows that $(x, y) \in r$. From just proved inclusion we have that $\sup \left[\sup [x)_{r}\right)_{r} \leq \sup [x)_{r}$, which means that $c_{r}\left(c_{r}(x)\right) \leq c_{r}(x)$. Finally, $c_{r} \in R T(A)$.

Now we can consider the complete lattice $(C l(A), \leq)$ of all closure operations defined on $(A, \leq)$. Obviously, $C l(A)=E x(A) \cap R T(A)$. Consider also the class $Q O r d_{\text {fun }}^{m o n}=R e f l_{f u n} \cap \operatorname{Trans}_{\text {fun }}^{\text {mon }}$ of all quasiorders (reflexive and transitive relations) $r$ defined on $A$ satisfying two conditions: (fun) and (mon). The following corollary follows immediately from Facts 3 and 5 .

Corollary 6. The correspondence $c \longmapsto r_{c}$, where for any $x, y \in A$, $(x, y) \in r_{c}$ iff $y \leq c(x)$, is an isomorphism of the lattices $(C l(A), \leq)$ and $\left(Q_{\text {Ord }}^{\text {mon }}, \subseteq\right)$. The correspondence $r \longmapsto c_{r}$, where for each $x \in A, c(x)=$ $\sup [x)_{r}$, is the inverse isomorphism of the lattices.

It turns out that class $Q O r d_{f u n}^{m o n}$ can be defined in a much simpler way. In order to do it, first let us notice two simple facts.

FACT 7. For any transitive relation $r$ on $A$, the conjuction of the conditions (mon) and reflexivity is equivalent to the inclusion $\leq \sim \subseteq r$, where $\leq \sim$ is the converse of the lattice order $\leq$. 
Proof. Assume that $r$ is transitive on $A$.

$(\Rightarrow)$ : Suppose that (mon) is satisfied by $r$ and $r$ is reflexive. Let $(x, y) \in$ $\leq \sim$, that is, $y \leq x$. Since $(y, y) \in r$, from (mon) it follows that $(x, y) \in r$. Therefore, $\leq \sim \subseteq r$.

$(\Leftarrow)$ : Assume that $\leq^{\sim} \subseteq r$ and let $(x, z) \in r$, and $x \leq y$. Then $(y, x) \in \leq \sim$. Consequently, $(y, x) \in r$ by the assumption. Then $(y, z) \in r$ due to transitivity of $r$. Thus condition (mon) holds true. Obviously $r$ is reflexive since $\leq$ is reflexive.

FACT 8. For any quasiorder $r$ on A satisfying (mon), the condition (fun) is equivalent to the following one: for each $x \in A, \sup [x)_{r} \in[x)_{r}$.

Proof. Let $r$ be any quasiorder satisfying (mon). The proof in the direction $(\Rightarrow)$ follows immediately due to (fun').

$(\Leftarrow)$ : Assume that for each $x \in A, \sup [x)_{r} \in[x)_{r}$. Take any $x \in A$. We are going to show that $[x)_{r}=\left(\sup [x)_{r}\right]_{\leq}$, actually that $\left(\sup [x)_{r}\right]_{\leq} \subseteq[x)_{r}$ since the converse inclusion always holds. So suppose that $y \leq \sup [x)_{r}$. Since $(y, y) \in r$ so from (mon), we have $\left(\sup [x)_{r}, y\right) \in r$. From the assumption it follows that $\left(x, \sup [x)_{r}\right) \in r$. Therefore, $(x, y) \in r$ due to transitivity of $r$.

Due to Facts 7, 8 the simple characteristic of $Q O r d_{f u n}^{m o n}$ follows.

COROLlary 9. QOrd $d_{\text {fun }}^{\text {mon }}$ is the class of all transitive relations $r$ on $A$ such that $\leq \sim \subseteq r$ and for each $x \in A, \sup [x)_{r} \in[x)_{r}$.

The class is a proper subclass of all quasiorders greater than $\leq \sim$ with respect to inclusion. Let us provide some examples.

Consider the complete lattice $(\langle 0,1\rangle, \leq)$ of all real numbers from the interval $\langle 0,1\rangle$. Then the relation $r=\langle 0,1\rangle^{2}-\{(x, 1): x \in\langle 0,1\rangle-\{1\}\}$ is a quasiorder on the set $\langle 0,1\rangle$ such that $\leq \sim \subseteq r$, however, it does not satisfy condition (fun) since $\sup [0)_{r}=1 \notin[0)_{r}$. Here, for any $x \in\langle 0,1\rangle-$ $\{1\},[x)_{r}=\langle 0,1\rangle-\{1\}$ and $[1)_{r}=\langle 0,1\rangle$.

Consider also the Boolean 4-element lattice $(A, \leq)$, where $A=\{0, a, b, 1\}$ and the relation $r=A^{2}-\{(0,1),(a, 1),(b, 1)\}$. Then $r$ is a quasiorder on $A$ such that $\leq \sim \subseteq r$, however, $\sup [0)_{r}=1 \notin[0)_{r}$ since $[0)_{r}=[a)_{r}=[b)_{r}=$ $\{0, a, b\}$ and $[1)_{r}=A$. 


\section{Syntactic characteristic of closure operations}

Let $(A, \leq)$ be any complete lattice and $r \subseteq A^{2}$ any binary relation defined on $A$. We say that an element $b \in A$ is closed on the relation $r$ iff for any $(x, y) \in r, x \leq b \Rightarrow y \leq b$.

FACT 10. For any $r \subseteq A^{2}$ the set $B_{r} \subseteq A$ of all closed elements on $r$ is a closure system of the lattice $(A, \leq)$.

Proof. Let $B \subseteq B_{r}$. We show that $\inf B$ is closed on $r$. So let $(x, y) \in r$ and $x \leq \inf B$. Then for each $b \in B, x \leq b$. It means that for each $b \in B, y \leq b$ since each element from $B$ is closed on $r$. Thus, $y \leq \inf B$.

Let $G: \wp\left(A^{2}\right) \longrightarrow C l(A)$ be a mapping assigning to each relation $r \subseteq A^{2}$ the closure operation determined by the closure system $B_{r}$, that is, for any $r \subseteq A^{2}$, and any $x \in A, G(r)(x)=\inf \left\{b \in B_{r}: x \leq b\right\}$. Conversely, let $F: C l(A) \longrightarrow \wp\left(A^{2}\right)$ be a map assigning to each closure operation $c$ the relation $r_{c}$ defined by $(x, y) \in r_{c}$ iff $y \leq c(x)$. In this way we reach a generalization of (4').

Proposition 11. $(F, G)$ is a residuated pair of mappings for the complete lattices $(C l(A), \leq),\left(\wp\left(A^{2}\right), \subseteq\right)$ such that for any $c \in C l(A), G(F(c))=c$.

Proof. Let $r_{1}, r_{2} \subseteq A^{2}$ and $r_{1} \subseteq r_{2}$. Then $B_{r_{2}} \subseteq B_{r_{1}}$. In result, given an $x \in A,\left\{b \in B_{r_{2}}: x \leq b\right\} \subseteq\left\{b \in B_{r_{1}}: x \leq b\right\}$. Hence $\inf \left\{b \in B_{r_{1}}\right.$ : $x \leq b\} \leq \inf \left\{b \in B_{r_{2}}: x \leq b\right\}$, that is, $G\left(r_{1}\right)(x) \leq G\left(r_{2}\right)(x)$; therefore $G\left(r_{1}\right) \leq G\left(r_{2}\right)$, which means that $G$ is monotone. Similarly, when for given $c_{1}, c_{2} \in C l(A), c_{1} \leq c_{2}$, we have that $(x, y) \in F\left(c_{1}\right)$ is equivalent to $y \leq c_{1}(x)$, for any $x, y \in A$. At the same time, $y \leq c_{1}(x)$ implies $y \leq c_{2}(x)$ and $y \leq c_{2}(x)$ is equivalent to $(x, y) \in F\left(c_{2}\right)$. So $F\left(c_{1}\right) \subseteq F\left(c_{2}\right)$ and thus $F$ is also monotone. Now we show that for any $r \subseteq A^{2}, r \subseteq F(G(r))$. So let $(x, y) \in r$. Then from the definition of the closure system $B_{r}$ it follows immediately that $y \leq \inf \left\{b \in B_{r}: x \leq b\right\}$ that is, $y \leq G(r)(x)$, which means that $(x, y) \in F(G(r))$. In order to show that for any closure operation $c$ of the lattice $(A, \leq), G(F(c))=c$, we prove that - given $c \in C l(A)$ and an $x \in A-\inf \left\{b \in B_{F(c)}: x \leq b\right\}=c(x)$. First, notice that for any $b \in A, b \in B_{F(c)}$ iff for any $z, y \in A(y \leq c(z) \& z \leq b \Rightarrow y \leq b)$. Obviously, for arbitrary $y, z \in A$, the condition $y \leq c(z) \& z \leq c(x) \Rightarrow y \leq$ $c(x)$ holds. Moreover, $x \leq c(x)$. Therefore, we have $c(x) \in\left\{b \in B_{F(c)}\right.$ : $x \leq b\}$. Consequently, $\inf \left\{b \in B_{F(c)}: x \leq b\right\} \leq c(x)$. In order to show 
the converse inequality, take any $b \in B_{F(c)}$ such that $x \leq b$. Then we have that for any $z, y \in A(y \leq c(z) \& z \leq b \Rightarrow y \leq b)$. So $c(x)$ and $x$ replace $y, z$, respectively, yielding $c(x) \leq c(x) \& x \leq b \Rightarrow c(x) \leq b$. From this it follows that $c(x) \leq b$, so $c(x)$ is a lower bound of the set $\left\{b \in B_{F(c)}: x \leq b\right\}$ in the lattice $(A, \leq)$; hence $c(x) \leq \inf \left\{b \in B_{F(c)}: x \leq b\right\}$.

From Proposition 11 it follows that the mapping $F$ is an isomorphism of the complete lattice $(C l(A), \leq)$ (of all open elements with respect to interior operation $F \circ G$ ) to the complete lattice of all closed elements with respect to closure operation $G \circ F$ defined on $\left(\wp\left(A^{2}\right), \subseteq\right)$. So the latter lattice is just a generalized counterpart of the lattice $(\operatorname{Relcons}(L), \subseteq)$ we have searched for. It coincides with the range of $F$ ordered by inclusion. According to Corollary 6, the map $F$ is an isomorphism of the lattices $(C l(A), \leq)$ and $\left(Q_{\text {Ord }}^{\text {mon }}, \subseteq\right)$. Obviously, $Q O r d_{f u n}^{\text {mon }} \subseteq \wp\left(A^{2}\right)$. So we have that the complete lattice $\left(Q O r d_{\text {fun }}^{\text {mon }}, \subseteq\right)$ plays the role of the lattice $(\operatorname{Relcons}(L), \subseteq)$. One may check this result proving the fact that the restriction of the mapping $G$ to the set $Q O r d_{\text {fun }}^{\text {mon }}$ coincides with the inverse isomorphism from Corollary 6.

Proposition 12. For any binary relation $r \in \operatorname{QOrd}_{\text {fun }}^{\text {mon }}$ and any $x \in A$, $G(r)(x)=\sup [x)_{r}$, that is, $G(r)=c_{r}$.

Proof. Suppose that $r \in Q O r d_{\text {fun }}^{\text {mon }}$ and let $x \in A$. We simply show that the element $\sup [x)_{r}$ is the greatest lower bound of the set $\left\{b \in B_{r}: x \leq b\right\}$. So at first take any $b \in B_{r}$ such that $x \leq b$. Then for any $z \in A((x, z) \in$ $r \& x \leq b \Rightarrow z \leq b)$. Now, take any $y \in[x)_{r}$, that is $(x, y) \in r$. So we obtain $y \leq b$. In this way, $\sup [x)_{r} \leq b$ since $y$ has been chosen arbitrarily. However, this means that $\sup [x)_{r}$ is a lower bound of the set $\left\{b \in B_{r}: x \leq b\right\}$ in the lattice $(A, \leq)$. Now assume that a $u \in A$ is such a lower bound, i.e., the following holds: $\forall b \in B_{r}(x \leq b \Rightarrow u \leq b)$. Thus, in order to show that $u \leq \sup [x)_{r}$, it is sufficient to prove that $\sup [x)_{r} \in B_{r}$ and $x \leq \sup [x)_{r}$. Notice that $x \leq \sup [x)_{r}$ follows from reflexivity of $r$ while $\sup [x)_{r} \in B_{r}$ follows from transitivity of $r$ and conditions (mon) and (fun). To show the latter, suppose that $(y, z) \in r$ and $y \leq \sup [x)_{r}$. Then $\left(\sup [x)_{r}, z\right) \in r$ from (mon), moreover, $\left(x, \sup [x)_{r}\right) \in r$ from (fun). Thus, $(x, z) \in r$ from transitivity of $r$, so $z \in[x)_{r}$ and, finally, $z \leq \sup [x)_{r}$.

In this way, our proposal of syntactic account of consequence operation defined on a lattice $(\wp(L), \subseteq)$, consists in replacing a consequence relation 
defined by conditions (r1)-(r3) with a transitive relation $\vdash \subseteq \wp(L) \times \wp(L)$ such that for any $X, Y \subseteq L, Y \subseteq X \Rightarrow X \vdash Y$ and $X \vdash \bigcup[X)_{r}$, where $[X)_{r}=\{Z \subseteq L: X \vdash Z\}$ (comp. Corollary 9). Then, having a relation of this kind, the corresponding consequence operation $C: \wp(L) \longrightarrow \wp(L)$ is defined by $C(X)=\bigcup[X)_{r}$. Conversely, starting from a consequence operation $C$, the corresponding relation $\vdash$ on $\wp(L)$ is defined by $X \vdash Y$ iff $Y \subseteq C(X)$.

\section{References}

[1] T. S. Blyth, Lattices and Ordered Algebraic Structures, Springer (2005).

[2] K. Denecke, M. Erné, S. L. Wismath (eds.), Galois Connections and Applications, Kluwer (2004).

[3] F. Domenach, B. Leclerc, Biclosed binary relations and Galois connections, Order 18 (2001), pp. 89-104.

[4] M. Erné, J. Koslowski, A. Melton, G. E. Strecker, A Primer on Galois Connections, Annals of the New York Academy of Sciences, vol. 704 (1993), pp. 103-125.

[5] D. J. Shoesmith, T. J. Smiley, Multiple-conclusion Logic, Cambridge University Press (1978).

[6] R. Wójcicki, Lectures on Propositional Calculi, Ossolineum (1984).

[7] R. Wójcicki, Theory of Logical Calculi. Basic Theory of Consequence Operations, Kluwer (1988).

[8] J. Zygmunt, An Essay in Matrix Semantics for Consequence Relations, Wydawnictwo Uniwersytetu Wrocławskiego, Wrocław (1984).

Department of Logic

University of Lodz

Lindleya $3 / 5$

90-131 Łódź

Poland

e-mail: marnowak@filozof.uni.lodz.pl 